\title{
Automation of a Versatile Crane (the LSMS) for Lunar Outpost Construction, Maintenance and Inspection
}

\author{
William R. Doggett, Carlos M. Roithmayr, John T. Dorsey, Thomas C. Jones \\ NASA Langley Research Center, Hampton Va 23662 \\ Haijun Shen, Hans Seywald \\ Analytical Mechanics Associates, Inc., Hampton Va 23666 \\ Bruce D. King \\ Lockheed Martin Mission Services, Hampton Va 23662 \\ and \\ Martin M. Mikulas \\ National Institute of Aerospace, Hampton Va 23662
}

Devices for lifting, translating and precisely placing payloads are critical for efficient Earth-based construction operations. Both recent and past studies have demonstrated that devices with similar functionality will be needed to support lunar outpost operations. Although several designs have been developed for Earth based applications, these devices lack unique design characteristics necessary for transport to and use on the harsh lunar surface. These design characteristics include: a) lightweight components, b) compact packaging for launch, c) automated deployment, d) simple in-field reconfiguration and repair, and e) support for tele-operated or automated operations. Also, because the cost to transport mass to the lunar surface is very high, the number of devices that can be dedicated to surface operations will be limited. Thus, in contrast to Earth-based construction, where many single-purpose devices dominate a construction site, a lunar outpost will require a limited number of versatile devices that provide operational benefit from initial construction through sustained operations.

The first generation test-bed of a new high performance device, the Lunar Surface Manipulation System (LSMS) has been designed, built and field tested. The LSMS has many unique features resulting in a mass efficient solution to payload handling on the lunar surface. Typically, the LSMS device mass is estimated at approximately $3 \%$ of the mass of the heaviest payload lifted at the tip, or $1.8 \%$ of the mass of the heaviest mass lifted at the elbow or mid-span of the boom for a high performance variant incorporating advanced structural components.

Initial operational capabilities of the LSMS were successfully demonstrated during field tests at Moses Lake, Washington using a tele-operated approach. Joint angle sensors have been developed for the LSMS to improve operator situational awareness. These same sensors provide the necessary information to support fully automated operations, greatly expanding the operational versatility of the LSMS. This paper develops the equations describing the forward and inverse relation between LSMS joint angles and Cartesian coordinates of the LSMS tip. These equations allow a variety of schemes to be used to maneuver the LSMS to optimize the maneuver. One such scheme will be described in detail that eliminates undesirable swinging of the payload at the conclusion of a maneuver, even when the payload is suspended from a passive rigid link. The swinging is undesirable when performing precision maneuvers, such as aligning an object for mating or positioning a camera. Use of the equations described here enables automated control of the LSMS greatly improving its operational versatility. 


\section{Nomenclature}

$\begin{array}{ll}R & =\text { Reach of LSMS } \\ \eta R & =\text { Height to shoulder, } 0 \leq \eta \leq 1 \\ \beta R & =\text { Distance from shoulder to elbow, } 0 \leq \beta \leq 1 \\ \text { LSMS } & =\text { Lunar Surface Manipulation System } \\ \theta_{1} & =\text { Waist joint angle } \\ \theta_{2} & =\text { Shoulder joint angle } \\ \theta_{3} & =\text { Elbow joint angle relative to arm } \\ \theta_{4} & =\text { Lifting link angle from the vertical } \\ \gamma_{\text {pitch }} & =\text { Wrist pitch angle } \\ \gamma_{\text {roll }} & =\text { Wrist roll angle } \\ \gamma_{\text {yaw }} & =\text { Wrist yaw angle }\end{array}$

\section{Introduction}

$\mathrm{D}$ evices for lifting, translating and precisely placing payloads are critical for efficient Earth-based construction operations. Both recent and past studies have demonstrated that devices with similar functionality will be needed to support lunar outpost operations. ${ }^{1,2}$ Lunar payloads include: a) prepackaged hardware and supplies that must be unloaded from landers and then accurately located at their operational site, b) sensor packages used for periodic inspection of landers, habitat surfaces, etc., and c) local materials such as regolith that require excavation and grading. ${ }^{3}$ Although several designs have been developed for Earth based applications, these devices lack unique design characteristics necessary for transport to and use on the harsh lunar surface. ${ }^{4}$ These design characteristics include: a) lightweight components, b) compact packaging for launch, c) automated deployment, d) simple in-field reconfiguration and repair, and e) support for tele-operated or automated operations. Also, because the cost to transport mass to the lunar surface is very high, the number of devices that can be dedicated to surface operations will be limited. Thus, in contrast to Earth-based construction, where many single-purpose devices dominate a construction site, a lunar outpost will require a limited number of versatile devices that provide operational benefit from initial construction through sustained operations.

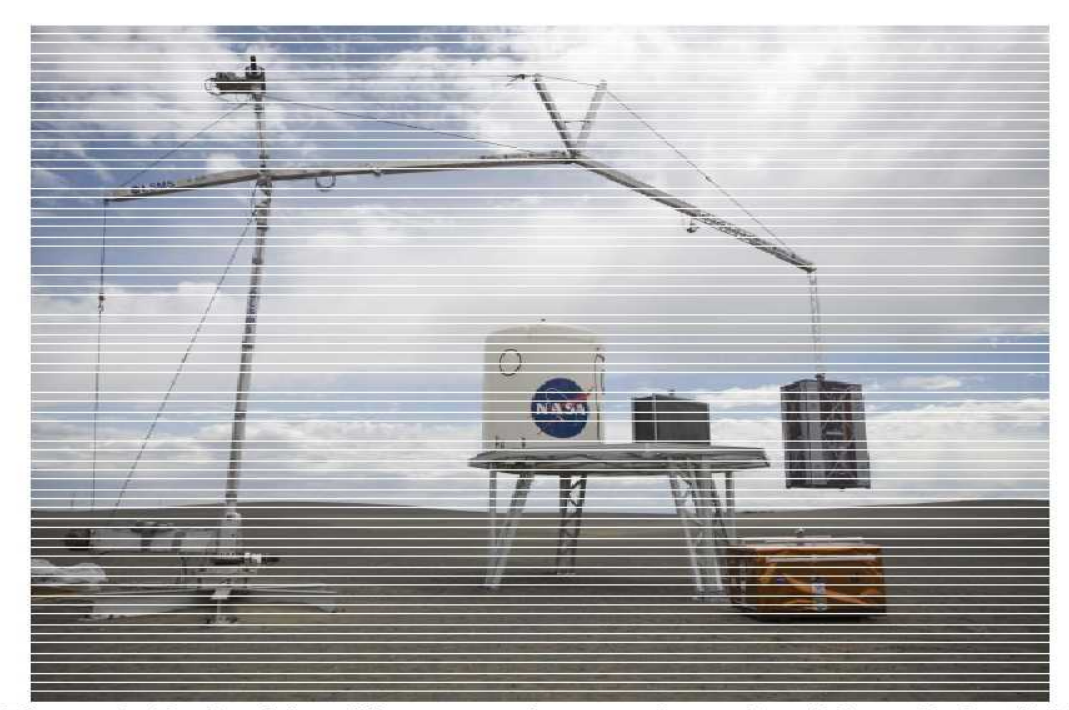

Figure 1. Payload handling operations performed at Moses Lake field
The first generation testbed of a new high performance device, the Lunar Surface Manipulation System (LSMS) has been designed and built. ${ }^{5}$ The LSMS has many unique features resulting in a mass efficient solution to payload handling on the lunar surface. Typically, the LSMS device mass is estimated at approximately $3 \%$ of the mass of the heaviest payload lifted at the tip, or $1.7 \%$ of the mass of the heaviest mass lifted at the elbow or mid-span of the boom for a high performance variant incorporating structural advanced During laboratory tests, the 
LSMS closely matched structural analysis predictions. Initial operational capabilities of the LSMS were successfully demonstrated during field tests at Moses Lake, Washington (Fig. 1) using a tele-operated approach. ${ }^{5}$

A natural enhancement to the current tele-operated (person in the loop) control is automated control. Automated control is necessary for several reasons including the ability to direct the LSMS from remote sites (for example the lunar outpost or earth) and to enable interaction with global planning tools to evaluate different concepts of operations. The joint angle sensors necessary to provide an operator with situational awareness during tele-operated maneuvers are directly applicable to automated control, thus no burden is placed on the system design to support automated control.

This paper will describe the design and installation of joint angle sensors to support automated operations and situational awareness for a remote operator. Then the equations for the forward and inverse kinematics necessary for automated control will be provided. The forward kinematic equations describe the location of the tip of the LSMS when the joint angles are known, the inverse kinematic equations are used to determine the joint angles necessary to position the tip at a desire location. The inverse kinematics allows direct translation from Cartesian goals to joint level commands to achieve those goals. For example, the inverse kinematics enable straight line Cartesian moves of the LSMS in either tip or potentially more useful camera defined frames providing intuitive motion control to the operator. A common scheme for describing robotic manipulators uses four parameters called the Denavit-Hartenburg parameters. The Denavit-Hartenburg parameters for the LSMS are provided in appendix A.

The forward and inverse kinematic equations allow a variety of schemes to be used to maneuver the LSMS to optimize the maneuver. One such scheme will be described in detail that eliminates undesirable swinging of the payload at the conclusion of a maneuver, even when the payload is suspended from a passive rigid link called a lifting link. This results in the lifting link and payload behaving like a double pendulum. It is possible to eliminate undesirable swinging motion at the conclusion of a maneuver using optimal control techniques. Three approaches have been investigated and are discussed in this paper, and numerical results obtained with each scheme are provided.

\section{LSMS Test-Bed Overview}

Figure 2 identifies the major components of the LSMS Test-Bed, and Table 1 provides the corresponding dimensions for the first generation test-bed constructed in 2008. Referring to the figure, the primary design drivers for the LSMS are the maximum reach, $R$, the shoulder height, $\eta R$ where $0 \leq \eta \leq 1$, and the maximum payload mass to be lifted at the wrist or elbow in lunar or Mars gravity. The LSMS design scales easily over a wide range of reaches and payload masses. Because of the efficient structural design, the total device mass for a device based on composite trusses is typically approximately $3 \%$ of the maximum load lifted at the wrist or $1.7 \%$ of the maximum load lifted at the elbow. Details on the scaling laws are provided in Refs. 6 and 7. The LSMS has 3 primary degrees of freedom, provided by revolute joints identified by the red dotted call-outs in the figure. Using the inertial frame identified in Fig. 2 and starting at the ground interface (or base) of the device, the first revolute joint is referred to as the waist joint and it provides a simple rotation about the positive $\mathrm{z}$ axis represented by the angle $\theta_{1}$ measured from the positive $\mathrm{x}$ axis. The second revolute joint is located at the top of the king post at a distance $\eta R(0 \leq \eta \leq 1)$ along the positive $\mathrm{z}$ axis. This joint is called the shoulder joint, and it provides a simple rotation about an axis parallel to positive y axis that is represented by the angle $\theta_{2}$ measured from a horizontal line passing through the shoulder. The third revolute joint, called the elbow joint, is positioned at a distance $\beta R(0 \leq \beta \leq 1)$ in the positive $\mathrm{x}$ direction from the shoulder. It enables a simple rotation about an axis parallel to the positive $\mathrm{y}$ axis that is represented by the angle $\theta_{3}$ measured from a line passing through the shoulder and elbow joints. The joints are actuated by electric motors. The electric motors drive hoists to actuate the shoulder and elbow while an electric motor drives the waist rotation directly. Finally, the wrist is located at the end of the forearm by continuing along positive $\mathrm{x}$ a distance $R(1-\beta)$. In the figure a lifting link, or rigid connection used to lift payloads is shown. The angle of the lifting link relative to the vertical is identified by $\theta_{4}$. 


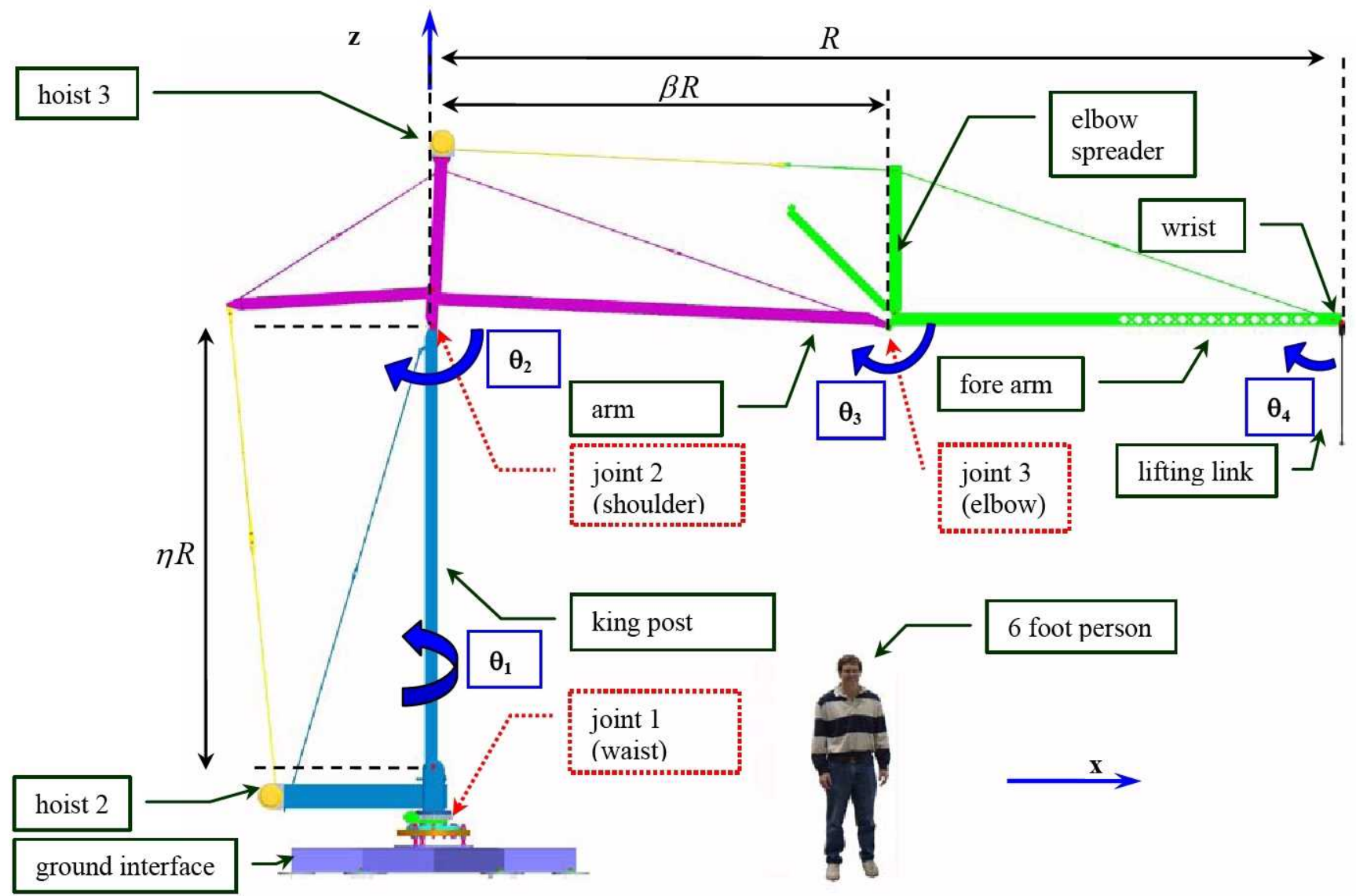

Figure 2. LSMS Naming Convention.

Table 1, LSMS Test-Bed Dimensions and Capability

\begin{tabular}{|c|ccccc|}
\hline & $\begin{array}{c}\text { Height } \\
(\eta R[\mathbf{m}])\end{array}$ & $\begin{array}{c}\text { Wrist Reach } \\
(\mathbf{R}[\mathbf{m}])\end{array}$ & $\begin{array}{c}\text { Elbow Reach } \\
(\beta R[\mathbf{m}])\end{array}$ & $\begin{array}{c}\text { Lunar Wrist } \\
\text { Capacity }([\mathrm{kg}])\end{array}$ & $\begin{array}{c}\text { Lunar Elbow } \\
\text { Capacity }([\mathrm{kg}])\end{array}$ \\
\hline Generation I & 3.75 & 7.5 & 3.76 & 1000 & 1765 \\
\hline
\end{tabular}

\section{Joint Angle Sensors for the LSMS}

Initial development of the LSMS focused on mechanical design of the test-bed with an eye toward automated operations in the future. Having successfully demonstrated the LSMS in the field in June of 08, the focus of 09 was to develop a sensing system for the joint angles of the LSMS as well as to develop the forward and inverse kinematics for the LSMS in preparation for automated control. Joint sensors were integrated to measure each active joint; waist, shoulder and elbow shown in Fig. 2. In each case a cam is affixed to one side of the joint and the sensor package is affixed to the other. The sensor package is based on a pair of Keyence GT series displacement sensors that measure the radial distance from the joint axis. This distance varies linearly with angle and is controlled by the profile on the cam. Two profiles were machined into each cam. This provides a redundant measurement of position and in addition, the difference between the 2 position measurements also varies linearly with angle providing a robust method to counteract eccentricity in the cam mounting. Figures 3 and 4 depict the details of the sensor mounting at the elbow. Figure 3 is a view from under the LSMS looking up in the positive $\mathrm{z}$ direction. A close up of the sensors and cam is shown in Fig. 4. Cabling to the sensors has been omitted from the figures for clarity. The sensors are mounted to the forearm via a bracket, the cam is affixed to the arm and rotates about the elbow joint pin. The bracket houses 2 plungers that transmit the radial displacement to the sensor heads while absorbing any transverse loads on the sensors. In addition, use of the plungers reduces the width of the cam. The mounting at the shoulder is very similar to that at the elbow and uses an identical cam. Figures 5 and 6 depict the mounting at the 
waist. The waist uses a larger cam, however the change in radial displacement is the same as the elbow and shoulder enabling the same sensor model to be used at all locations.

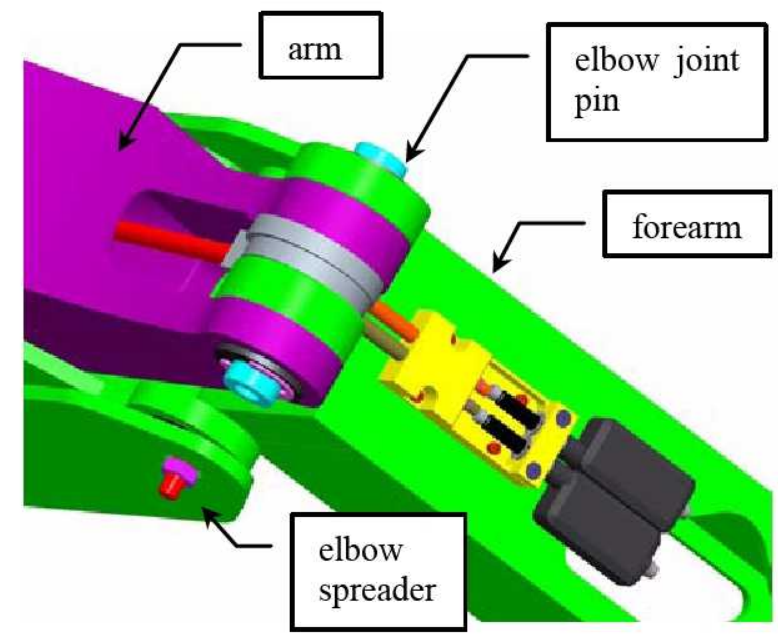

Figure 3. Bottom View of Elbow Sensors.

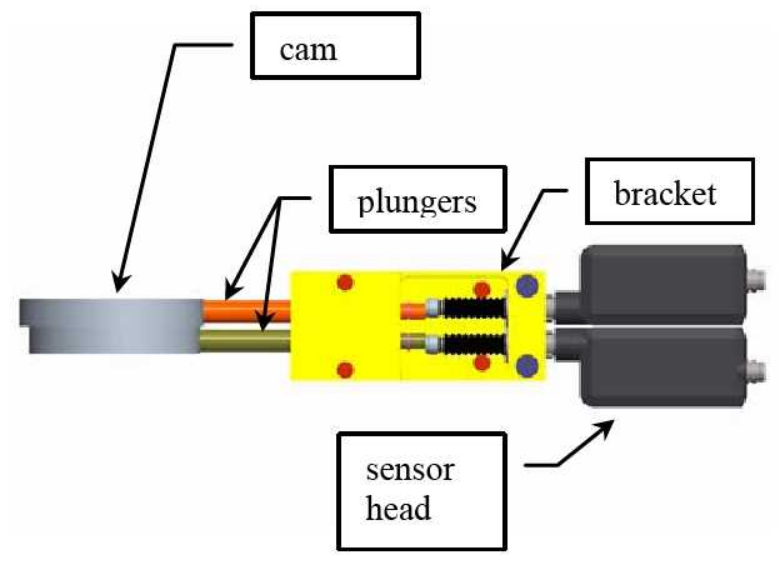

Figure 4. Elbow and Shoulder Sensor System.

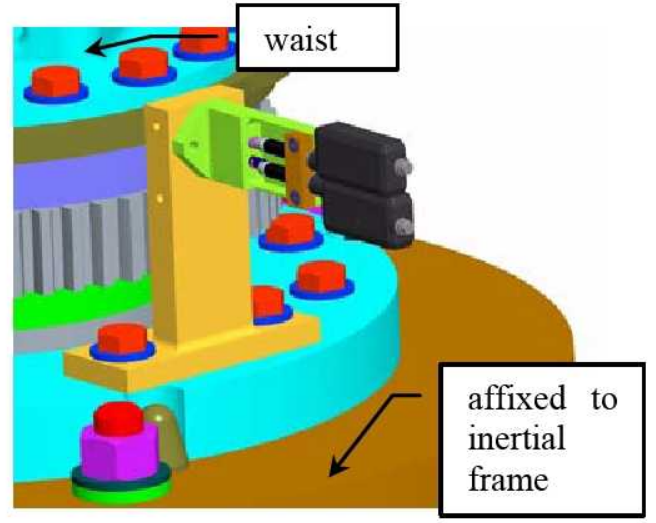

Figure 5. Side View of Waist Sensors.

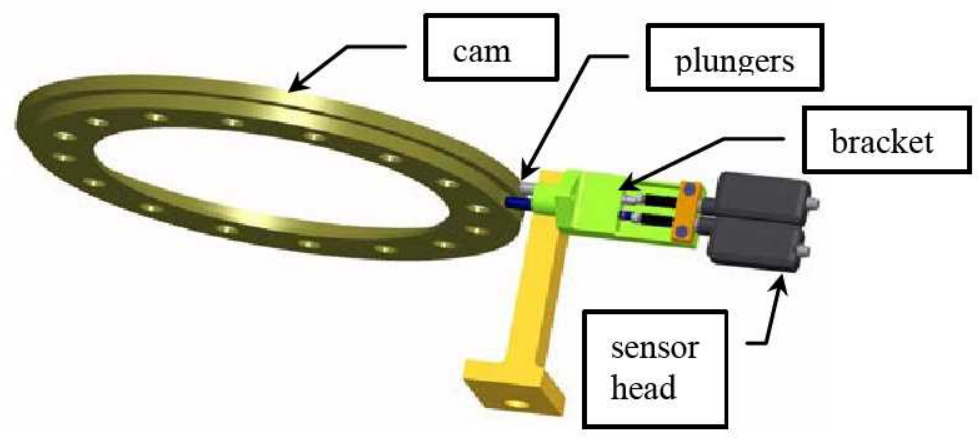

Figure 6. Waist Cam and Sensor System.

\section{Forward Kinematics of the LSMS}

This section will develop the equations that describe the position and orientation of each link with respect to the inertial frame when the joint angles are known. Of particular importance is a set of equations that describe the location of the wrist in the inertial frame when the joint angles are known. The previous section described one method for measurement of the joint angles of the LSMS. Three transformations can be used to relate the relative position and orientations of the links of the LSMS, and a fourth transformation describes the relationship between the wrist and the elbow frame. The transformations will be defined following the notation provided by Craig using the local reference frames identified in Fig. $7 .^{8}$

In Craig's notation, a $4 \times 4$ homogeneous transform is used to describe the relative position and orientation of two frames. Equation (1) depicts a homogenous transform describing the position and orientation of frame B in frame A as indicated by the leading sub and super scripts of $T$. The upper left $3 \times 3$ of the $4 \times 4$ homogenous transform is a rotation matrix, the columns of which are unit vectors defining the directions of the axes of $\mathrm{B}$ in frame A, i.e. if $\left[\hat{a}_{1}, \hat{a}_{2}, \hat{a}_{3}\right]$ are three unit vectors fixed in frame $\mathrm{A}$ and $\left[\hat{b}_{1}, \hat{b}_{2}, \hat{b}_{3}\right]$ are three unit vectors fixed in $\mathrm{B}$, then $r_{s t}=\hat{a}_{s} \cdot \hat{b}_{t},(s, t=1,2,3)$. The vector $\left[p_{x}, p_{y}, p_{z}\right]^{T}$ in Eq. (1) locates the origin of frame $\mathrm{B}$ in frame A. 


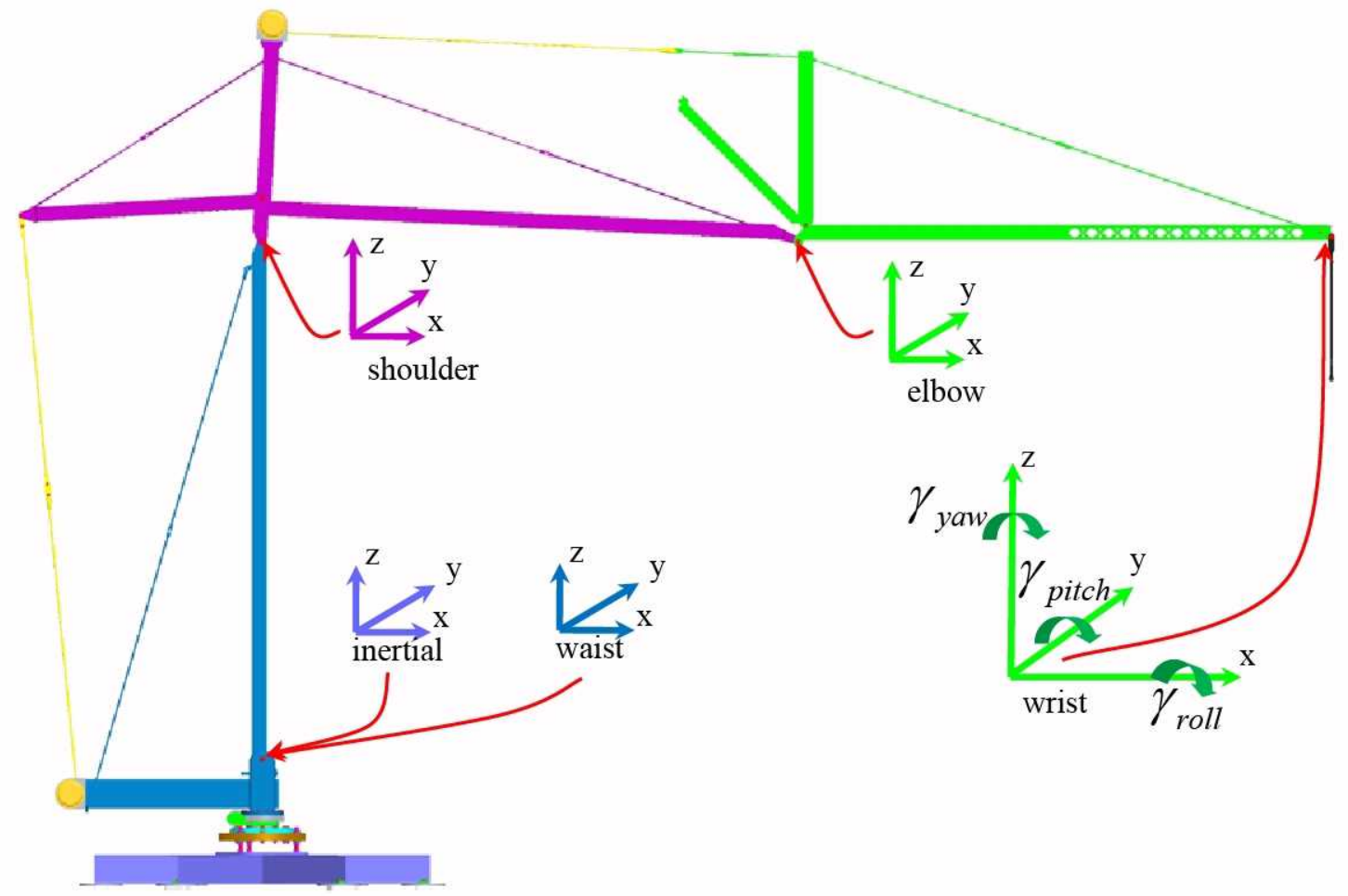

Figure 7. Local Reference Frames.

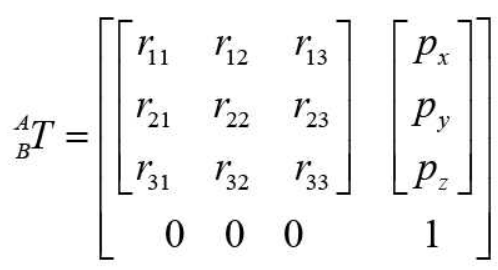

Referring to Fig. 7, local reference frames are affixed to each link, i.e. each group of components that move as a unit are shown with a similar color in the figure and this color is also used for the axes of the reference frame. The local frames shown in the figure do not follow the Denavit-Hartenberg convention due to consistency with other analysis efforts. For completeness, a set of Denavit-Hartenberg parameters along with corresponding reference frames has been included in appendix A. Starting at the base, or inertial frame, the first local frame is the waist frame upon which the blue components move, followed by the shoulder frame about which the magenta components rotate, then the elbow frame in which the green components rotate as a unit, and finally the wrist frame. The lifting link shown in black rotates relative to the wrist about a pin parallel to the y axis of the wrist frame. The lifting link is a passive link. Using the reference frames, it is straightforward to describe the location of the wrist with respect to the inertial frame. Starting at the base of the LSMS and working toward the wrist, the relation between the local reference frames are given by:

$$
\underset{\text { waist }}{\text { inertial }} T=\left[\begin{array}{cccc}
\cos \left(\theta_{1}\right) & -\sin \left(\theta_{1}\right) & 0 & 0 \\
\sin \left(\theta_{1}\right) & \cos \left(\theta_{1}\right) & 0 & 0 \\
0 & 0 & 1 & 0 \\
0 & 0 & 0 & 1
\end{array}\right]
$$




$$
\begin{aligned}
\underset{\text { waist }}{\text { shoulder }} T & =\left[\begin{array}{cccc}
\cos \left(\theta_{2}\right) & 0 & \sin \left(\theta_{2}\right) & 0 \\
0 & 1 & 0 & 0 \\
-\sin \left(\theta_{2}\right) & 0 & \cos \left(\theta_{2}\right) & \eta R \\
0 & 0 & 0 & 1
\end{array}\right] \\
{ }_{\text {elbow }}^{\text {shoulder }} T= & {\left[\begin{array}{cccc}
\cos \left(\theta_{3}\right) & 0 & \sin \left(\theta_{3}\right) & \beta R \\
0 & 1 & 0 & 0 \\
-\sin \left(\theta_{3}\right) & 0 & \cos \left(\theta_{3}\right) & 0 \\
0 & 0 & 0 & 1
\end{array}\right] } \\
{ }_{\text {elbow }}^{\text {wrist }} T= & {\left[\begin{array}{ccccc}
1 & 0 & 0 & R(1-\beta) \\
0 & 1 & 0 & 0 \\
0 & 0 & 1 & 0 \\
0 & 0 & 0 & 1
\end{array}\right] }
\end{aligned}
$$

Then the forward kinematics of the LSMS, i.e. the definition of the wrist in the inertial frame, is given by

$$
\begin{aligned}
& { }_{\text {wrist }}^{\text {inertial }} T={ }_{\text {waist }}^{\text {inertial }} T_{\text {shoulder }} T_{\text {elbow }}^{\text {waist }} T_{\text {wrist }}^{\text {shoulder }} T \text { or } \\
& \underset{\text { inrist }}{\text { witial }} T=\left[\begin{array}{cccc}
\operatorname{Cos}\left(\theta_{1}\right) \operatorname{Cos}\left(\theta_{2}+\theta_{3}\right) & -\operatorname{Sin}\left(\theta_{1}\right) & \operatorname{Cos}\left(\theta_{1}\right) \operatorname{Sin}\left(\theta_{2}+\theta_{3}\right) & R \operatorname{Cos}\left(\theta_{1}\right)\left[\beta \operatorname{Cos}\left(\theta_{2}\right)+(1-\beta) \operatorname{Cos}\left(\theta_{2}+\theta_{3}\right)\right] \\
\operatorname{Cos}\left(\theta_{2}+\theta_{3}\right) \operatorname{Sin}\left(\theta_{1}\right) & \operatorname{Cos}\left(\theta_{1}\right) & \operatorname{Sin}\left(\theta_{1}\right) \operatorname{Sin}\left(\theta_{2}+\theta_{3}\right) & R \operatorname{Sin}\left(\theta_{1}\right)\left[\beta \operatorname{Cos}\left(\theta_{2}\right)+(1-\beta) \operatorname{Cos}\left(\theta_{2}+\theta_{3}\right)\right] \\
-\operatorname{Sin}\left(\theta_{2}+\theta_{3}\right) & 0 & \operatorname{Cos}\left(\theta_{2}+\theta_{3}\right) & R\left[\eta-\beta \operatorname{Sin}\left(\theta_{2}\right)-(1-\beta) \operatorname{Sin}\left(\theta_{2}+\theta_{3}\right)\right] \\
0 & 0 & 0 & 1
\end{array}\right]
\end{aligned}
$$

Equation (6) is commonly used to transform a target location in the wrist frame into a target location in the inertial frame. This transformation occurs during operator or sensor driven motion control where the camera or sensor system is attached to the wrist. The inverse kinematics developed in the next section are then used to convert the target location into corresponding joint angles which can be used to drive the motors of the robot so that the wrist arrives at the desired target location. It is straight forward to locate any other frame of Fig. 7 relative to the inertial frame by a similar process, thus with knowledge of the joint angles, the location of all the links are precisely known.

\section{Inverse Kinematics of the LSMS}

The inverse kinematic problem is generally much more difficult than the forward kinematic problem for a serial manipulator, however because of the design simplicity of the LSMS, the inverse solution is not complex. The inverse kinematic problem is to compute the set of joint angles, the joint vector $\left[\theta_{1}, \theta_{2}, \theta_{3}\right]^{T}$, that locates the wrist at a target position. Note the LSMS has 3 degrees of freedom, thus only three of six coordinates describing the position and orientation of the wrist may be specified. In the following, two sets of target coordinates will be specified. The first is the orientation of the wrist with respect to inertial frame, i.e. $\left[\gamma_{\text {roll }}, \gamma_{\text {yaw }}, \gamma_{\text {pitch }}\right]^{T}$. This set of relations can be determined by inspection and they are:

$$
\begin{aligned}
& \gamma_{\text {pitch }}=\theta_{2}+\theta_{3} \\
& \gamma_{\text {yaw }}=\theta_{1} \\
& \gamma_{\text {roll }} \text { (no control authority) }
\end{aligned}
$$

Note, if a particular operation requires wrist roll, then it can easily be accommodated by an appropriately designed end-effector or tool attached at the wrist. 
The second set of three target coordinates will be taken to be the position of the wrist in Cartesian space relative to the inertial frame, i.e. $\left[t_{x}, t_{y}, t_{z}\right]^{T}$. It is possible to solve for the explicit position of the tip in terms of the joint angles by selecting the wrist position vector from Eq. (6) and setting it equal to the desired target position, i.e.

$$
\begin{aligned}
t_{x} & =R \cos \left(\theta_{1}\right)\left[\beta \cos \left(\theta_{2}\right)+(1-\beta) \cos \left(\theta_{2}+\theta_{3}\right)\right] \\
t_{y} & =R \sin \left(\theta_{1}\right)\left[\beta \cos \left(\theta_{2}\right)+(1-\beta) \cos \left(\theta_{2}+\theta_{3}\right)\right] \\
t_{z} & =R\left[\eta-\beta \sin \left(\theta_{2}\right)-(1-\beta) \sin \left(\theta_{2}+\theta_{3}\right)\right]
\end{aligned}
$$

While the equations appear simple, the solution is lengthy. These equations were solved using Mathematica and the corresponding script commands are given in appendix B. Only the solution for $\theta_{1}$ is tractable and given by Eq. (10) below. Equation (10) agrees with intuition based on a top view looking down the $\mathrm{z}$ axis of the inertial frame.

$$
\theta_{1}=\operatorname{ATAN2}\left(t_{y}, t_{x}\right)
$$

Multiple solutions for a specified target position exist corresponding to location of the elbow joint above or below a line from the wrist to shoulder as depicted in Fig. 8 . These two configurations are called elbow up and elbow down respectively. In the figure the elbow up configuration is shown ghosted. When the LSMS reaches full extension, i.e. $\theta_{3}=0$, it is necessary to select which configuration to pursue for the current maneuver. This is managed by the control software.

\section{Ending a Maneuver with Payload at Rest}

The mechanical system of interest, sans the payload, is described in Sec. II. As discussed there, a lifting link is attached to the forearm at the wrist by means of a revolute joint. The lower end of the lifting link is used in grappling a

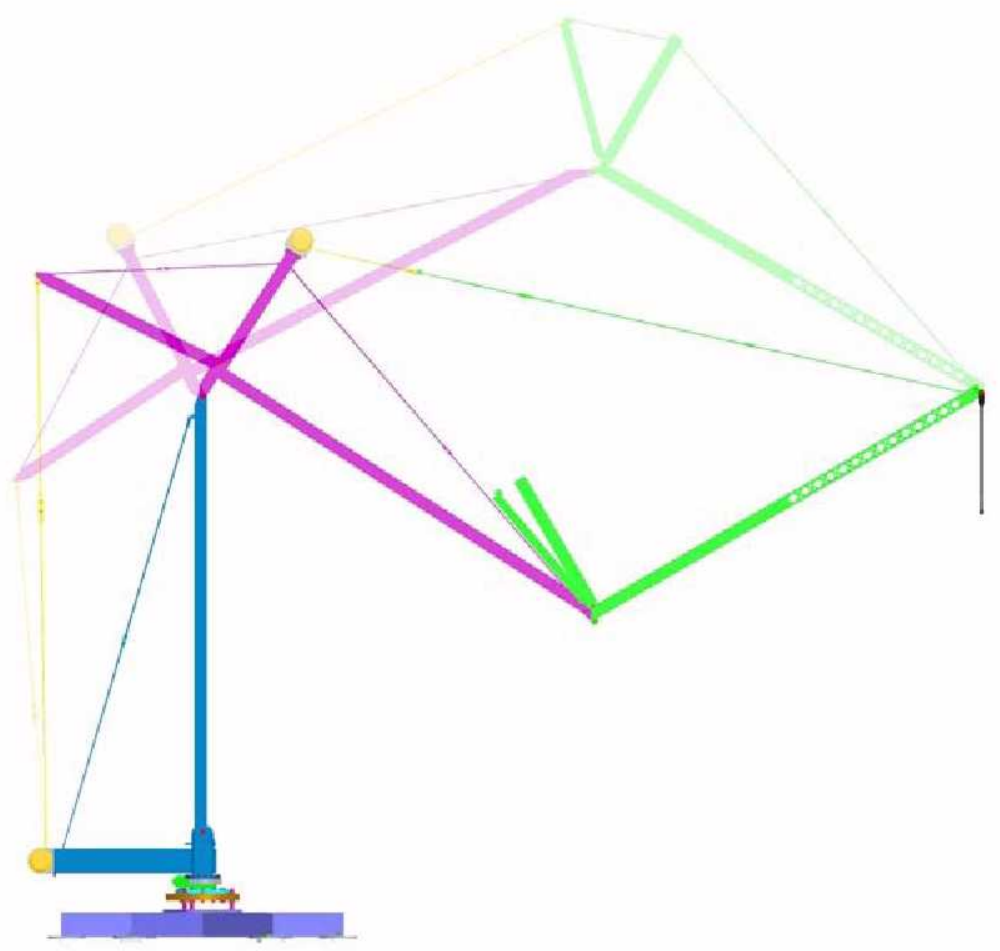

Figure 8. Two Solutions for a Wrist Location: Elbow Up vs. Down payload, and this connection is also modeled as a revolute joint. The two revolute joints connected to the lifting link have axes that are parallel to each other. Simple rotation of the payload relative to the lifting link is described by an angle $\theta_{5}$. The lifting link and payload thus have the configuration of a double pendulum, which is suspended from the forearm.

No motors are placed at the revolute joints connected to either end of the lifting link; hence, motion of the king post, arm, and forearm can result in oscillations of the lifting link and payload. Such swinging motion of the payload is undesirable for two reasons. First, it makes precise placement of the payload difficult; the payload may collide with other objects in close proximity to the intended final position. Depending on the amplitude of the oscillations and the amount of damping in the two revolute joints, the time required for the swinging to be damped 
out may represent a significant and unwanted delay in operations. Second, structural vibrations in the LSMS could be induced by swinging motion of the payload.

These considerations serve as motivation to find a way of maneuvering the LSMS and payload so that swinging of the lifting link and payload are eliminated at the conclusion of the maneuver. The problem of bringing the payload to rest when the maneuver ends is amenable to solution through the use of techniques of optimal control.

\section{A. Equations of Motion}

In order to solve an optimal control problem, one must have available a set of first-order differential equations governing the "state" of the system to be controlled. In other words, the kinematical differential equations and the dynamical equations of motion of the system are required. For this task, we employ the method of Kane as discussed in Refs. 9 and 10. The method involves choosing motion variables, also known as generalized speeds. In the case of a serial manipulator consisting of bodies fastened together by $n$ revolute joints, the time derivatives of the joint angles $\dot{\theta}_{r}(r=1, \ldots, n)$ are natural choices for motion variables, denoted here by $\omega_{r}$. The following relationships serve as definitions of the motion variables and as kinematical differential equations.

$$
\dot{\theta}_{r}=\omega_{r} \quad(r=1, \ldots, n)
$$

\section{(Error! Bookmark not defined.11)}

It is expedient to regard $m$ of the revolute joints $(m \leq n)$ as driven by motors that exert couples whose torques have directions parallel to the joint axes, and magnitudes that are the absolute values of the scalars $u_{r}(r=1, \ldots, m)$. Each body belonging to the manipulator is subject to gravitational force, considered to be applied at the body's mass center. The software AUTOLEV discussed in Ref. 10 makes use of computer algebra to derive explicit analytical dynamical equations of motion, given here in functional form as

$$
\dot{\omega}_{r}=g_{r}\left(\theta_{1}, \ldots, \theta_{n}, \omega_{1}, \ldots, \omega_{n}, u_{1}, \ldots, u_{m}, t\right) \quad(r=1, \ldots, n)
$$

where $t$ denotes time.

The system under consideration consists of five bodies (king post, arm, forearm, lifting link, and payload) and five revolute joints; thus, $n=5$. One motor is used to drive each of three joints (waist joint, shoulder joint, and elbow joint); therefore, $m=3$. For the purposes of this study, all bodies are considered to be rigid; appendix C contains the mass, inertia scalars, and mass center position for each of the five bodies, as well as position information for each of the five joints.

\section{B. Review of Optimal Control Problem Formulation}

The problem of bringing the manipulator payload to rest at the conclusion of a maneuver can be formulated as a problem in optimal control. As discussed (for example) in Ref. 11, the objective is to determine the functions $u_{1}(t), \ldots, u_{m}(t)$ that minimize (or maximize) a performance index $J$ expressed as

$$
J=\phi\left[x\left(t_{f}\right)\right]+\int_{t_{0}}^{t_{f}} L(x, u, t) d t
$$

subject to nonlinear differential equations of state

$$
\dot{x}=f(x, u, t)
$$

and subject to terminal constraints 


$$
\Psi\left[x\left(t_{f}\right)\right]=0
$$

In the case of a serial manipulator, $\theta_{1}, \ldots, \theta_{n}, \omega_{1}, \ldots, \omega_{n}$ constitute the elements of the state $x$. The differential equations (14) are simply a collection of Eqs. (11) and (12). In this paper we concern ourselves with problems for which initial values of all elements of the state, $\boldsymbol{x}\left(t_{0}\right)$, are specified. Functions $\Psi_{r}\left\lfloor x\left(t_{f}\right)\right\rfloor=0(r=$ $1, \ldots, q)$ of the state at the conclusion of the maneuver, $x\left(t_{f}\right)$, can be specified, where $q \leq 2 n$ for $L \neq 0$. Such requirements are often referred to as terminal constraints, and are represented by Eq. (15). A requirement that the manipulator and payload be in a certain configuration at the end of the maneuver is expressed with the relationships

$$
\Psi_{r}\left[x\left(t_{f}\right)\right]=\theta_{r}\left(t_{f}\right)-\bar{\theta}_{r}=0 \quad(r=1, \ldots, n)
$$

where $\bar{\theta}_{r}$ are specified constants. Similarly, a requirement that the payload and each body belonging to the manipulator be motionless at the final time can be stated as

$$
\Psi_{n+r}\left\lfloor x\left(t_{f}\right)\right\rfloor=\omega_{r}\left(t_{f}\right)=0 \quad(r=1, \ldots, n)
$$

Thus, in the problem of interest, $q=2 n$.

In what follows, the problem of bringing the payload to rest at the end of the LSMS maneuver is solved with

$$
\phi\left\lfloor x\left(t_{f}\right)\right\rfloor=0
$$

\section{Discontinuous Control}

The system under consideration is at rest in an inertial reference frame when $\omega_{r}=0$ and $\dot{\omega}_{r}=0(r=1, \ldots, 5)$. The condition of rest is possible when $\theta_{1}, \theta_{2}$, and $\theta_{3}$ take on any values whatsoever, and when $\theta_{4}=\theta_{5}=0$. Certain values of $u_{1}, u_{2}$, and $u_{3}$ are of course required; the axis of the waist joint is vertical, so it is readily seen that rest can occur with $u_{1}=0$, but $u_{2}$ and $u_{3}$ are in general not zero. The motors that control the shoulder joint and the elbow joint are designed such that they are able to prevent motion at the joint when no electric current is

applied. For example, a motor containing a worm gear can function in this manner. Thus, motor torque can be produced either by application of current or by the action of a brake; the first type of torque should be included in the function $L$ of Eq. (13), but the brake torque should not be. Such an approach renders $u_{2}$ discontinuous when included in $L$; it vanishes when $\omega_{2}=0$ but takes on a nonzero value otherwise. The same holds true for $u_{3}$. This complicates the formulation of the optimal control problem and makes numerical solution more difficult than it would be otherwise, as discussed presently.

\section{Numerical Solution of the Optimal Control Problem}

Methods for obtaining a numerical solution to an optimal control problem are categorized either as indirect, or direct.

Indirect methods involve solving the well-known Euler-Lagrange equations within the framework of a two-point (or multi-point) boundary value problem as discussed, for example, in Ref. 11. Prior to treating the LSMS discussed in this paper, methods given in Refs. 11 and 12 were applied to simplified models that did not include revolute joints at the shoulder or elbow. First, the payload was represented by a simple pendulum suspended from a cart translating along the outstretched arm, and no waist joint was included in the model. Subsequent models included waist joint motion, and a universal joint rather than a revolute joint was used to attach the pendulum to the cart. Encouraging results were obtained in these cases. However, the indirect methods failed when applied to the model presented 
here. Unfortunately, there are widely recognized shortcomings of the indirect method, including the requirement for good initial guesses of the time histories of states and costates, a small convergence radius, and so on.

On the other hand, the direct method does not involve the costates, and deals with the states and controls only. In this method, the states and/or controls are discretized in some manner. The system differential equations, the performance index, and the constraints are approximated by some numerical methods using the discrete values of the states and/or controls. In the end, the optimal control problem is transcribed into a nonlinear program with the discrete states and/or controls being the decision variables. The nonlinear program is then solved with a standard optimization technique. The direct method is advantageous over the indirect method in that it does not require a priori knowledge of the solution to start the process, and often it converges to an acceptable solution starting with only a rough guess. However, because of the discretization, the solution obtained with the direct method is not considered as accurate as the one obtained with the indirect method. Never-the-less, for practical purposes the former can be considered a good representation of the real solution when enough discrete nodes are used. The failure to obtain solutions for this problem with the indirect method leads us to employ the direct method.

EZopt ${ }^{1}$ is used to obtain the numerical solutions presented in this paper. EZopt is a software package that solves optimal control problems using the direct method. The entire time interval from $t_{0}$ to $t_{f}$ is divided into small segments by discrete nodes, the number and locations of which are set by the user. A collocation discretization scheme is used; states and controls are discretized at each node, with controls held constant between two neighboring nodes. (EZopt also allows differential inclusion.) The midpoint method is used to solve the differential equations over the interval between two neighboring nodes. The large-scale sparse optimizer SNOPT discussed in Ref. 13 is used to solve the resulting nonlinear program for the discrete state and control variables.

As mentioned previously, AUTOLEV is used to obtain algebraic expressions for the differential equations of state (14). In addition, the software is employed to obtain expressions for the partial derivatives $\partial / \partial x$ and $\partial f / \partial u$ needed to effect a numerical solution. AUTOLEV is instructed to write FORTRAN code containing the necessary expressions, and the code is used by EZOPT.

\section{Optimal Control Results}

Numerical results are presented in this section for three cases, each of which treats the brake mechanism differently. The results are presented in the order of increasing fidelity in the solutions. In the first case, the brake is not considered in the formulation; thus, all torques are supplied by the actuators. The second case incorporates the brake by using a smooth approximation of the step function as a mathematical model for brake engagement and disengagement. This approach involves numerical difficulties and turns out to be impractical, but it provides a significant amount of insight into how the joints and links behave with the brakes present. Information regarding the timing and duration of brake engagement is particularly helpful. The third case builds upon this information, and uses a phased approach to obtain a more precise problem formulation. In the third approach the entire maneuver is divided into phases, or intermittent time intervals during which the brakes are engaged or disengaged. During a phase when the brakes are engaged, explicit constraints are placed on the joint angular speeds so that they remain zero, and the torques are not penalized in the performance index. The phases are pieced together with the conditions that the states are continuous at the switching times between two neighboring phases. As formulated, the phased approach treats the brake as intended and thus leads to a more precise solution than the second case where the approximate model of the brake is used.

${ }^{1}$ Developed by Analytical Mechanics Associates, Inc. under a NASA SBIR contract.

11

American Institute of Aeronautics and Astronautics 
For all three cases, the following initial and final joint angles are considered.

Table 2. Initial and final joint angles

\begin{tabular}{|c||c|c|}
\hline$r$ & $\begin{array}{c}\theta_{r}\left(t_{0}\right) \\
(\mathrm{deg})\end{array}$ & $\begin{array}{c}\theta_{r}\left(t_{f}\right) \\
(\mathrm{deg})\end{array}$ \\
\hline 1 & 0 & 60 \\
\hline 2 & 20 & 60 \\
\hline 3 & -20 & -60 \\
\hline 4 & 0 & 0 \\
\hline 5 & 0 & 0 \\
\hline
\end{tabular}

The initial and final joint speeds are all zero. The maneuver time $t_{f}$ is 60 seconds. The angular speeds $\omega_{r}(t)$ are bounded by $\pm 10 \mathrm{deg} / \mathrm{s}$ for $r=1,2,3$, and by $\pm 20 \mathrm{deg} / \mathrm{s}$ for $r=4,5$. The motor torques $u_{r}(t)$ are bounded by \pm 3260 $\mathrm{Nm}$ for $r=1$, by $\pm 14620 \mathrm{Nm}$ for $r=2$, and by $\pm 6520 \mathrm{Nm}$ for $r=3$.

\section{A. Results Without Brakes}

As can be seen by inspecting the hardware design, a tremendous amount of torque must be applied at joints 2 and 3 to counter the moment due to gravity acting on the links and the payload. Without the brakes, the sole sources of torque are the electric motors at the active joints. In this approach, the integrand in Eq. (13) takes on the following form,

$$
L(x, u, t)=\frac{1}{2} u_{1}^{2}(t)+\frac{1}{2} u_{2}^{2}(t)+\frac{1}{2} u_{3}^{2}(t)
$$

Therefore, an energy-saving maneuver would try to reduce the torques at these joints as much as possible. One way of achieving this is to reduce the moment arms to the mass centers where the gravitational forces act. This strategy is evident in the numerical results, with the joint angles and actuator torques shown in Fig. 9, and the angular speeds shown in Fig. 10. The solution is obtained with 201 equally-distanced nodes. It is seen from the time histories of $\theta_{2}$, $\theta_{3}, \theta_{4}$, and $\theta_{5}$ that the robot quickly drops the links outboard of the shoulder so that they hang down from the shoulder, maintains that configuration for as long as possible, and then heads toward the desired final configuration. In the hanging configuration the moments of the gravitational forces about the joints vanish, and no torque is required at joints 2 and 3. Also, the moment of inertia of the entire system about the vertical axis is minimized, which in turn minimizes the torque required to turn the waist joint. As seen in the torque plot, the torques at joints 2 and 3 are close to zero during the middle section of the maneuver when the links are kept close to the hanging configuration.

\section{B. Results With Brakes}

Unfortunately, the hanging configuration is not likely to be a practical one. The range of motion at the shoulder joint will not permit this configuration because the payload and forearm will make contact with the ground. Therefore, torque will always be required at the shoulder and elbow joints to counteract gravity, whether the joints are moving or not. As discussed previously, each motor contains a brake that applies torque to prevent motion at the joint in the absence of electrical power. Two techniques for treating these brakes have been considered, and numerical results obtained with each technique are now presented.

\section{B.1 A solution using an approximate model of the brake}

The brake is a mechanically favorable design, but it presents a steep challenge to the optimal control problem formulation because with the brake the torque from the electric motor becomes a discontinuous function of the joint speed. Since the electric motors are engaged only when joints 2 and 3 are moved, the torques $u_{2}$ and $u_{3}$ should not be penalized in the performance index when $\omega_{2}=0$ and $\omega_{3}=0$, respectively. That is, the integrand of the performance index in Eq. (19) can be written as 


$$
L(x, u, t)=\frac{1}{2} u_{1}^{2}(t)+\frac{1}{2}\left[z\left(\omega_{2}\right) \cdot u_{2}(t)\right]^{2}+\frac{1}{2}\left[z\left(\omega_{3}\right) \cdot u_{3}(t)\right]^{2}
$$

where $z(\cdot)$ is a step function. However, the new performance index is a discontinuous and non-smooth function of $\omega_{2}$ and $\omega_{3}$, which cannot be handled within the EZopt environment. To circumvent this difficulty, a smooth approximation of the step function is used. That is, the step functions in Eq. (20) are replaced with

$$
\begin{aligned}
& \bar{Z}\left(\omega_{2}\right)=\operatorname{atan}\left(c \cdot \omega_{2}\right) /(\pi / 2) \\
& \bar{Z}\left(\omega_{3}\right)=\operatorname{atan}\left(c \cdot \omega_{3}\right) /(\pi / 2)
\end{aligned}
$$

and

where the coefficient $c$ controls the steepness of the function $\bar{z}(\cdot)$ rising from 0 to $1 . c=100$ is used to obtain the numerical results shown here.

The numerical results are illustrated in Figures 11 and 12. The solution is obtained with 201 equally-distanced nodes. From the time histories of the joint angles and angular speeds, it can be seen that the boundary conditions are satisfied. Because the brake is incorporated in the problem formulation, the robot does not go through the vertically hanging configuration. Instead, the time histories of $\theta_{2}$ and $\theta_{3}$ indicate the existence of periods when joint 2 or joint 3 moves extremely slowly. For all practical purposes, the two joints during these periods can be considered motionless. However, the joint speeds $\omega_{2}$ and $\omega_{3}$ exhibit chattering behavior during these time intervals. This is due to the numerical difficulties brought on by the steep gradients of the functions $\bar{z}(\cdot)$ at very low joint speed. In addition, the solution consists of too many such intervals (some are very short) when joints 2 and 3 are held motionless (at least 7 for joint 2 and 15 for joint 3 ), which could cause the robot to stop and go too frequently to be considered practical.

\section{B.2 Precise solution with a phased approach.}

The previous solution may not be numerically and practically sound, but it provides tremendous insight into how the links and joints behave when the brakes are included. For example, it reveals how many times and when the active joints may move or be held motionless. With this knowledge, a phased approach can be taken, and the brakes can be modeled precisely.

Based on the plot of $\theta_{2}$ in Fig. 11, the motion of joint 2 can be divided into three phases. The first phase is from 0 to 38 seconds, during which the joint is held fixed by the brake. The second phase spans from 38 to 52 seconds, and the electric motor is engaged to move the joint. In the last phase, from 52 to 60 seconds, the joint is again held still by the brake. Similar behavior can be seen in the plot of $\theta_{3}$ in Fig. 11.

In the numerical results in this section, the entire robot maneuver is divided into six phases. The time intervals and behaviors of $\theta_{2}$ and $\theta_{3}$ in each phase are summarized in Table 3. The time intervals of the phases are only an initial guess, and the switching times between phases are adjusted by the optimization process. During the phases when $\theta_{2}$ or $\theta_{3}$ is to be held constant, explicit constraints such as $\omega_{2}=0$ or $\omega_{3}=0$ are enforced, and thus, the joint torques during these phases are not penalized in the performance index at all. The initial and final conditions are enforced at the beginning of phase 1 and the end of phase 6, respectively. The phases are pieced together by the condition that the states remain continuous across the switching time between two consecutive phases. The number of nodes for each phase is also shown in Table 3 . Within each phase, the nodes are equally distanced.

Table 3. Characteristics of each phase.

\begin{tabular}{|l|l|l|l|l|l|l|}
\hline Phases & 1 & 2 & 3 & 4 & 5 & 6 \\
\hline $\begin{array}{l}\text { Guessed time interval } \\
\text { (seconds) }\end{array}$ & $0-22$ & $22-38$ & $38-42$ & $42-44$ & $44-52$ & $52-60$ \\
\hline$\theta_{2}$ constant? & Yes & Yes & No & No & No & Yes \\
\hline$\theta_{3}$ constant? & No & Yes & No & Yes & No & No \\
\hline Number of nodes & 201 & 601 & 21 & 56 & 26 & 101 \\
\hline
\end{tabular}


The numerical results are shown in Figures 13 and 14. It can be seen that the length of each phase is changed from the initial guess. The boundary conditions are satisfied; i.e., all joints and the payload start and end at rest. Unlike the approximate solution in the previous subsection, there is no chattering behavior of the angular speeds of joints 2 and 3 during the intervals when the joint angles are held constant. The torque plot shows that actuator torques $u_{2}$ and $u_{3}$ are applied only when the joints 2 and 3 are in motion. Finally, this solution is also more practical than the approximate solution because it has much fewer stop-and-go maneuvers.

It should be pointed out that the solution obtained with the second approach is indispensible in the sense that without it there is no better way to know a priori how many phases to have and when the phases switch in the phased approach. On the other hand, the solution from the phased approach should not be considered the best solution because the number of phases cannot be adjusted by the optimization process. However, the advantage of the phased approach is that the user controls the number of phases and the number of stop-and-go maneuvers for the active joints. 

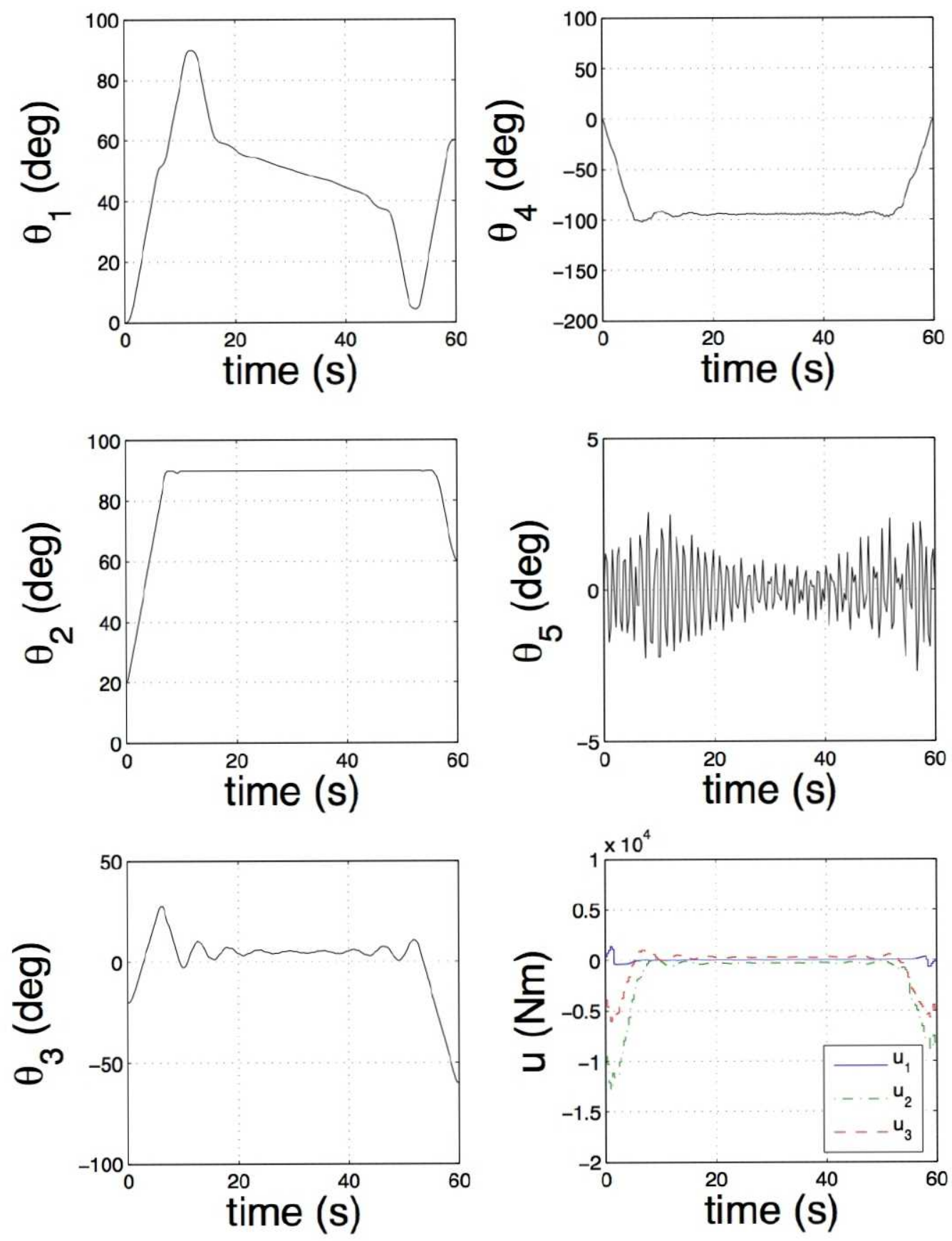

Figure 9. Joint angles and actuator torques when no brakes are used. 

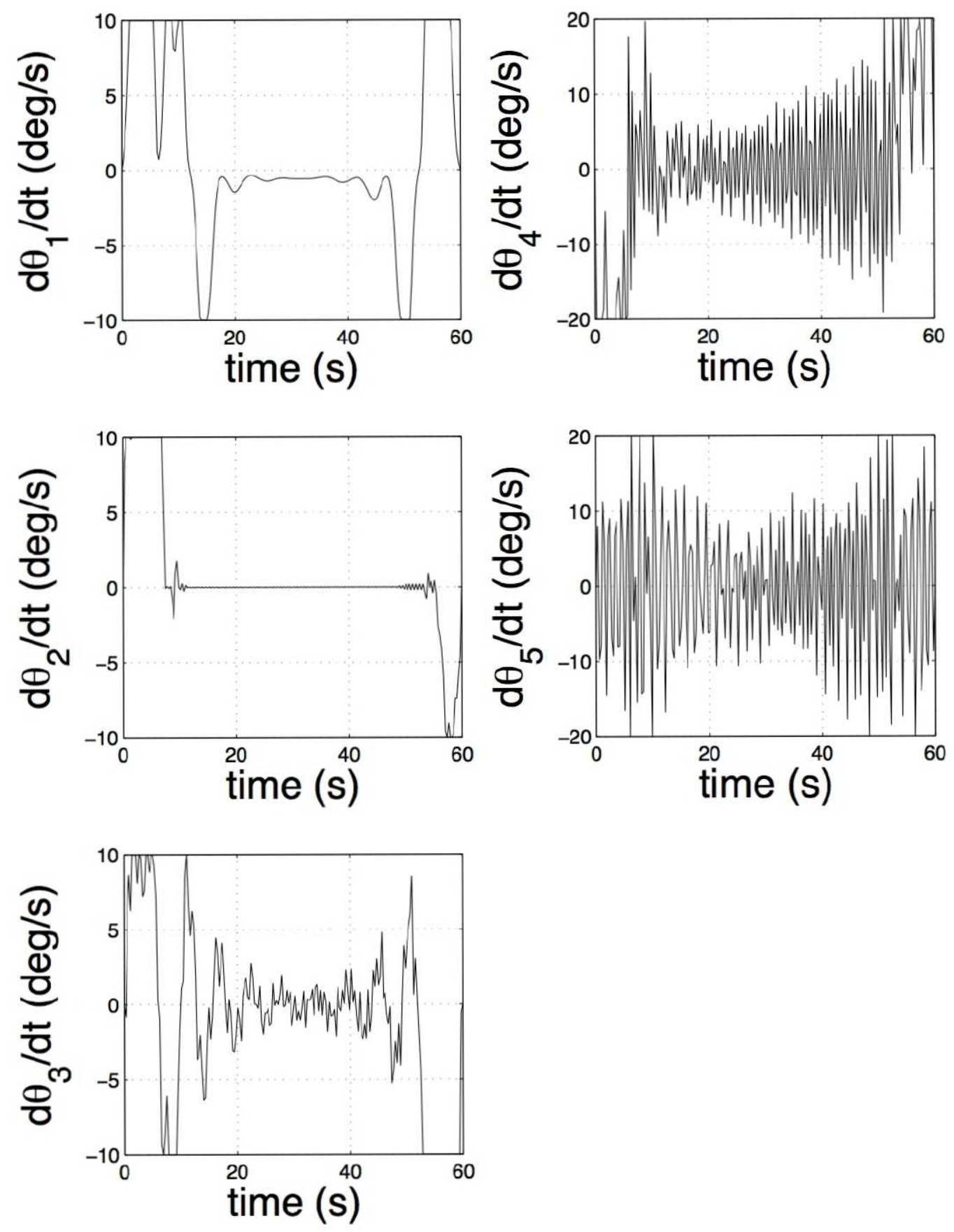

Figure 10. Angular speeds when no brakes are used. 

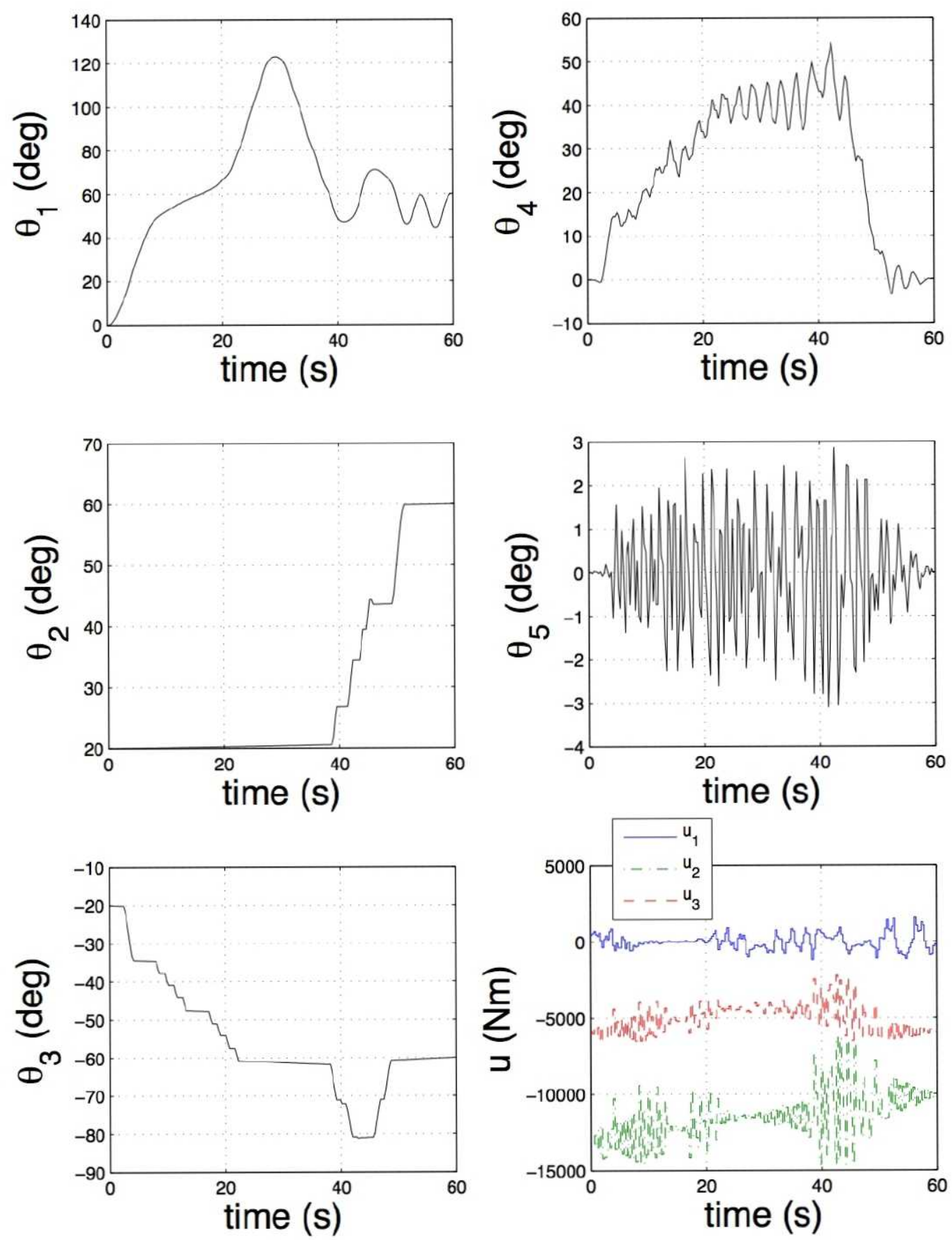

Figure 11. Joint angles and actuator torques when a smoothed step function is used. 

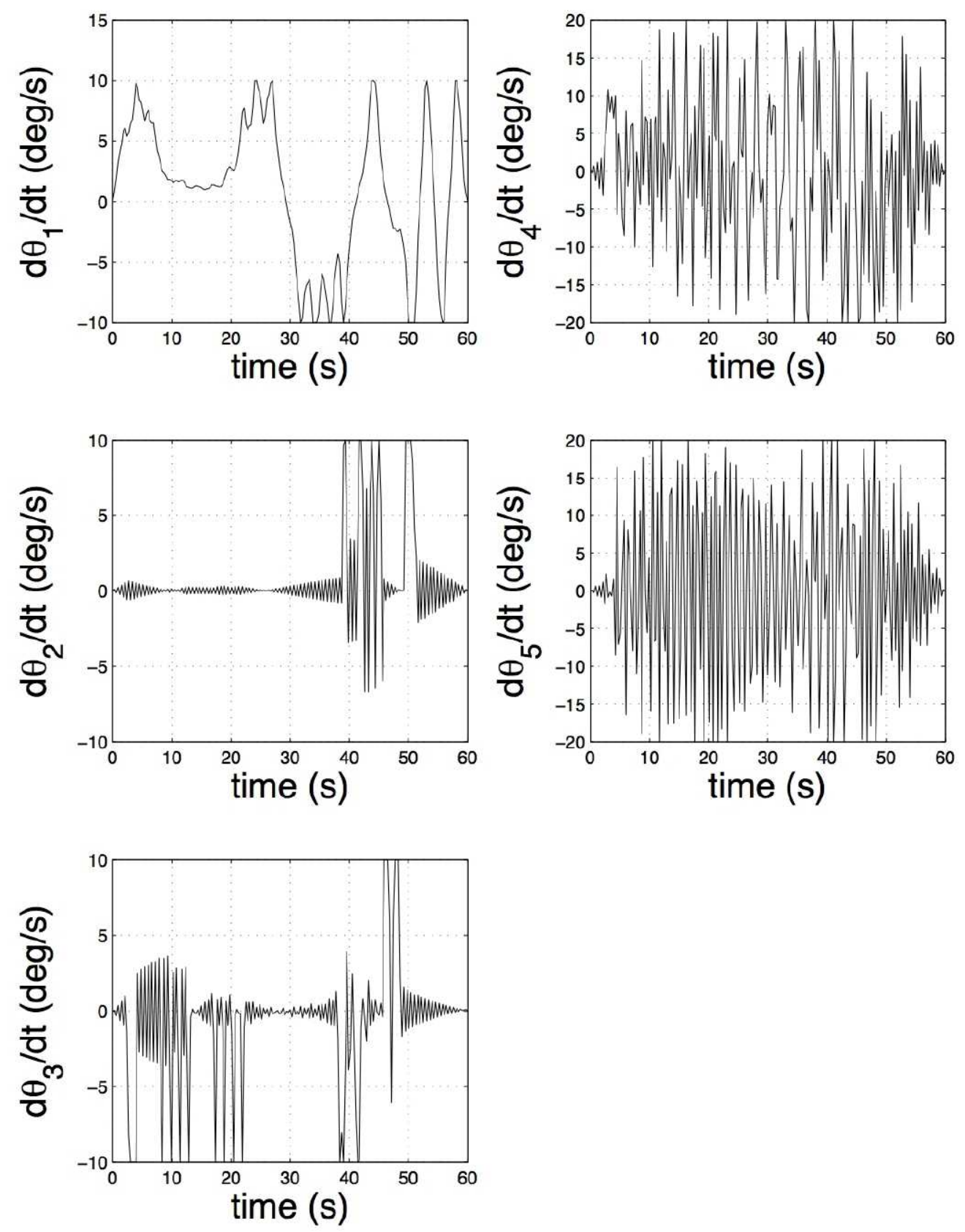

Figure 12. Angular speeds when a smoothed step function is used. 

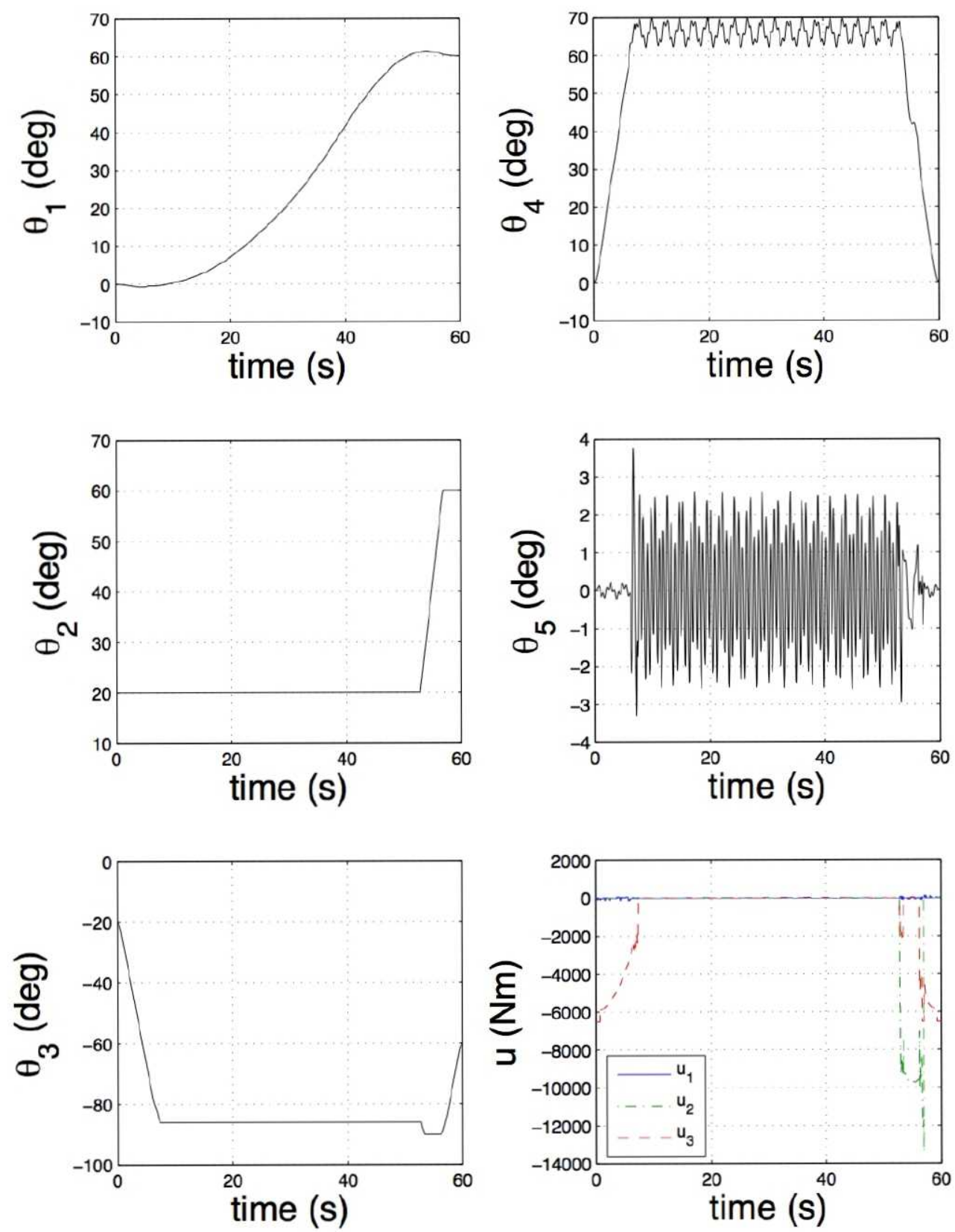

Figure 13. Joint angles and actuator torques with a 6-phase approach. 

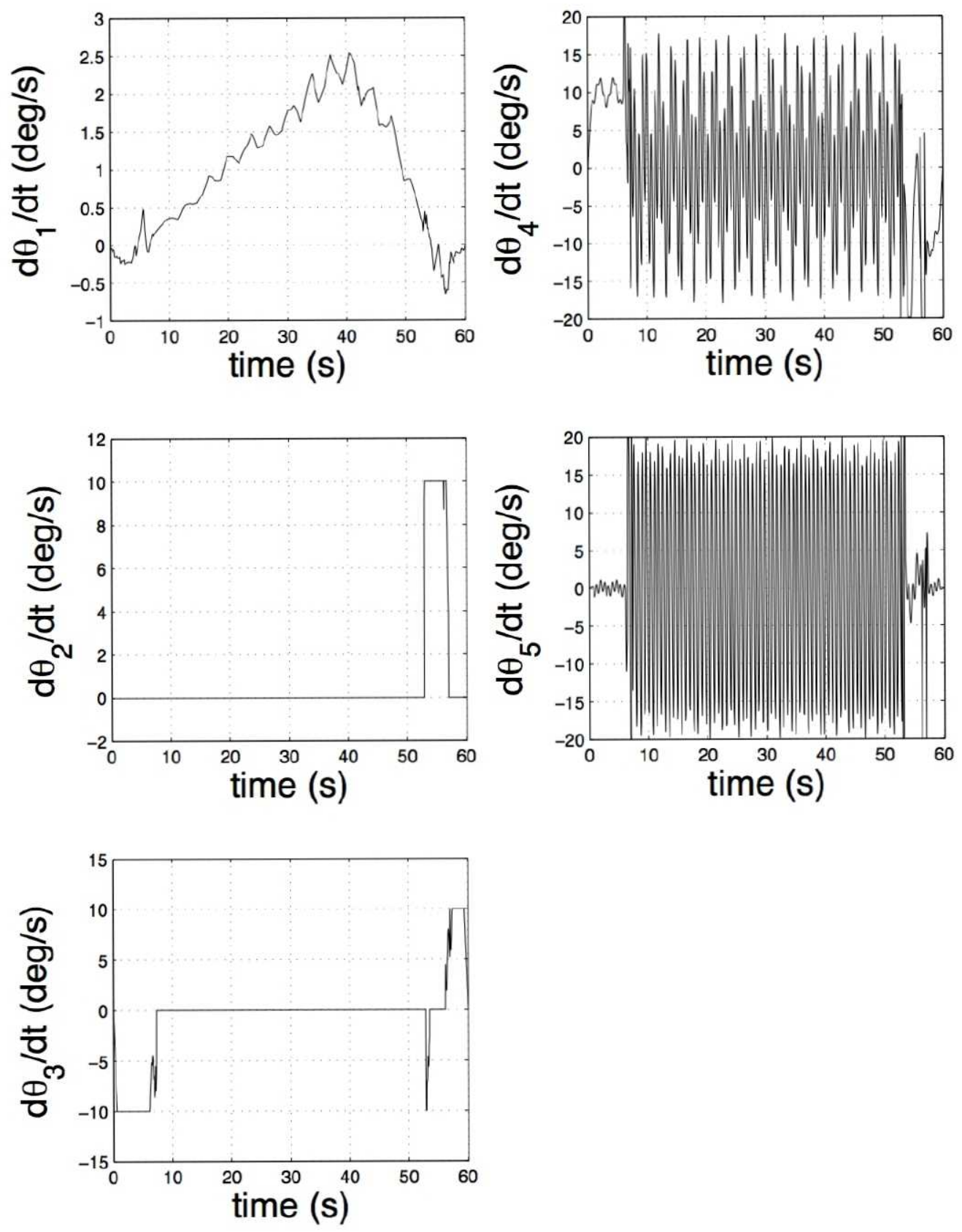

Figure 14. Angular speeds with a 6-phase approach. 


\section{Closing Comments}

A natural enhancement to the current tele-operated (person in the loop) control is automated control. Automated control is necessary for several reasons including the ability to direct the LSMS from remote sites (for example the lunar outpost or earth) and to enable interaction with global planning tools to evaluate different concepts of operations. The joint angle sensors necessary to provide an operator with situational awareness during tele-operated maneuvers are directly applicable to automated control, thus no burden is placed on the system design to support automated control.

Detail of the design and installation of joint angle sensors to support automated operations and situational awareness for remote operator were discussed. Then the forward kinematics were developed which can be used to locate the wrist in inertial coordinates when the joint angles are known. Using these equations a procedure was provided to obtain the inverse kinematic equations using Mathematica. The inverse kinematic equations allow direct translation from Cartesian goals in the inertial frame to joint level commands to achieve those goals. For example, the inverse kinematics enable Cartesian moves of the LSMS in either tip or potentially more useful camera defined frames providing intuitive motion control to the operator.

The forward and inverse kinematic equations allow a variety of schemes to be used to maneuver the LSMS to optimize the maneuver. One such scheme will be described in detail that eliminates undesirable swinging of the payload at the conclusion of a maneuver. Three approaches involving optimal control techniques are discussed, and numerical results obtained with each approach are provided. Of the three, the most promising approach accounts for a feature of a joint motor that allows it to serve as a brake in the absence of electrical power. Torque applied by the brake is not penalized in the performance index, but torque applied by the electric motor is penalized. The entire maneuver is divided into several phases during which, individually, the brakes at the shoulder and elbow joints either are, or are not, engaged. Using this approach, joint trajectories can be identified that eliminate the undesirable swing of the payload at the conclusion of the maneuver, enabling precision control of the payload using the LSMS. 


\section{Appendix A: Denavit Hartenburg Notation for LSMS}

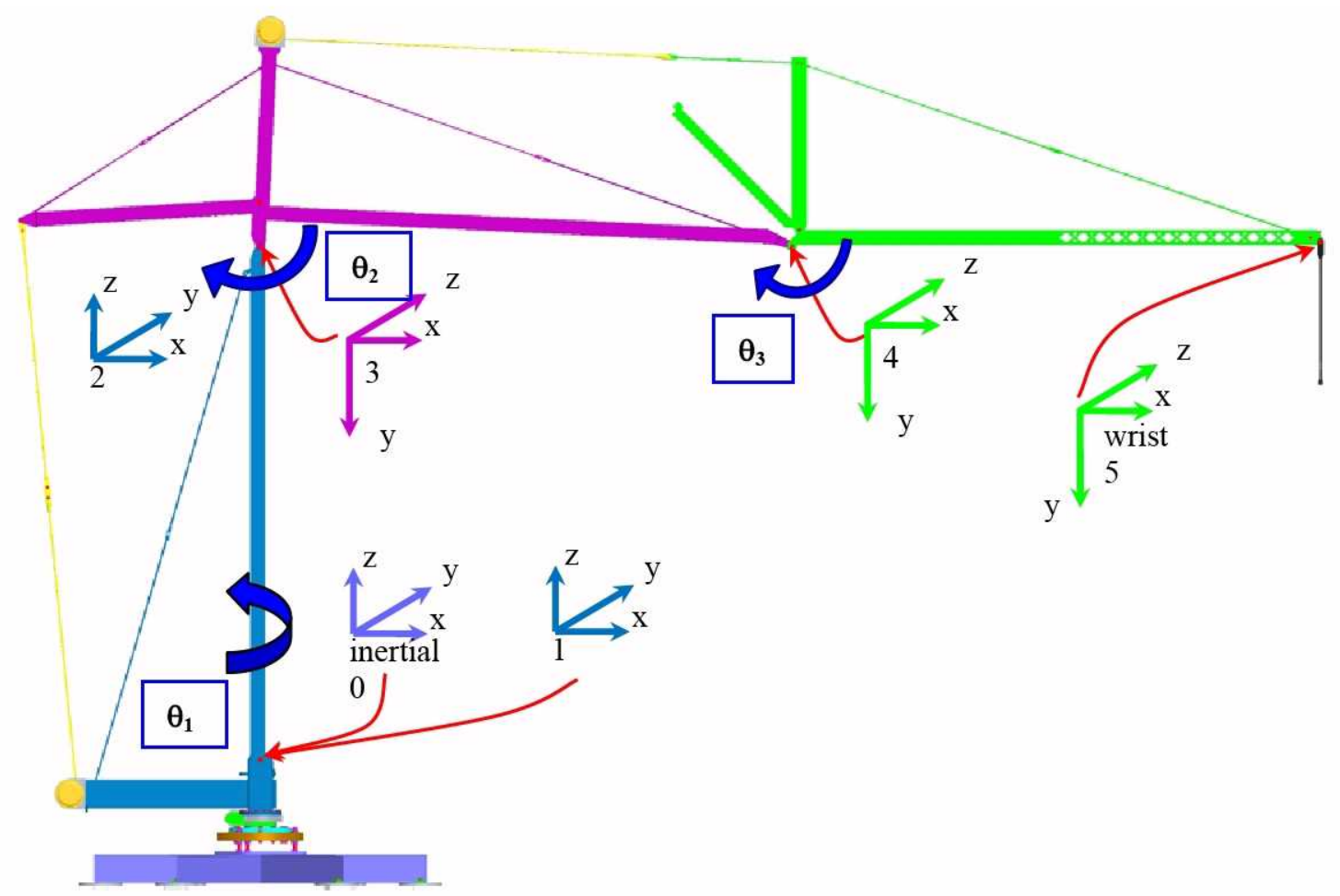

Figure 15. Local Reference Frames for Denavit-Hartenburg Parameter Definition.

The purpose of this appendix is to describe a set of the Denavit-Hartenburg parameters for the LSMS. The description of these parameters will follow the notation given by Craig. ${ }^{8}$ Denavit-Hartenburg parameters are a reduced set of parameters used to describe the relationship between links in a robotic manipulator. Four parameters are used to describe the relative location of two frames. They are:

link twist $\alpha_{i}$ = the angle between $\hat{Z}_{i \text { and }} \hat{Z}_{i+1}$ measured about $\hat{X}_{i}$,

link length $a_{i}=$ the distance from $\hat{Z}_{i}$ to $\hat{Z}_{i+1}$ measured along $\hat{X}_{i}$,

joint angle $\theta_{i}=$ the angle between $\hat{X}_{i-1}$ to $\hat{X}_{i \text { measured about }} \hat{Z}_{i \text {, and }}$

link offset $d_{i}=$ the distance from $\hat{X}_{i-1}$ to $\hat{X}_{i \text { measured along }} \hat{Z}_{i}$.

where $\hat{X}_{i}$ and $\hat{Z}_{i}$ are unit vectors in the ith $^{\text {th }}$ coordinate frame. Using these definitions the homogenous transform describing frame $i$ in frame $i-1$ is given by

$$
{ }_{i}^{i-1} T=\left[\begin{array}{cccc}
\cos \left(\theta_{i}\right) & -\sin \left(\theta_{i}\right) & 0 & a_{i-1} \\
\sin \left(\theta_{i}\right) \cos \left(\alpha_{i-1}\right) & \cos \left(\theta_{i}\right) \cos \left(\alpha_{i-1}\right) & -\sin \left(\alpha_{i-1}\right) & -\sin \left(\alpha_{i-1}\right) d_{i} \\
\sin \left(\theta_{i}\right) \sin \left(\alpha_{i-1}\right) & \cos \left(\theta_{i}\right) \sin \left(\alpha_{i-1}\right) & \cos \left(\alpha_{i-1}\right) & \cos \left(\alpha_{i-1}\right) d \\
0 & 0 & 0 & 1
\end{array}\right] .
$$

Referring to Fig. 15 the DH parameters are: 
Table 4, Denavit-Hartenburg parameters for LSMS.

\begin{tabular}{|c||c|c|c|c|}
\hline $\mathrm{i}$ & $\alpha_{i-1}$ & $a_{i-1}$ & $\theta_{i}$ & $d_{i}$ \\
\hline \hline 1(waist) & 0 & 0 & $\theta_{1}$ & 0 \\
\hline 2 & 0 & 0 & 0 & $\eta R$ \\
\hline 3 & $-\frac{\pi}{2}$ & 0 & $\theta_{2}$ & 0 \\
4 & 0 & $R \beta$ & $\theta_{3}$ & 0 \\
\hline 5 (wrist) & 0 & $R(1-\beta)$ & 0 & 0 \\
\hline
\end{tabular}




\section{Appendix B: Mathematica Code to Generate Inverse Kinematic Solutions}

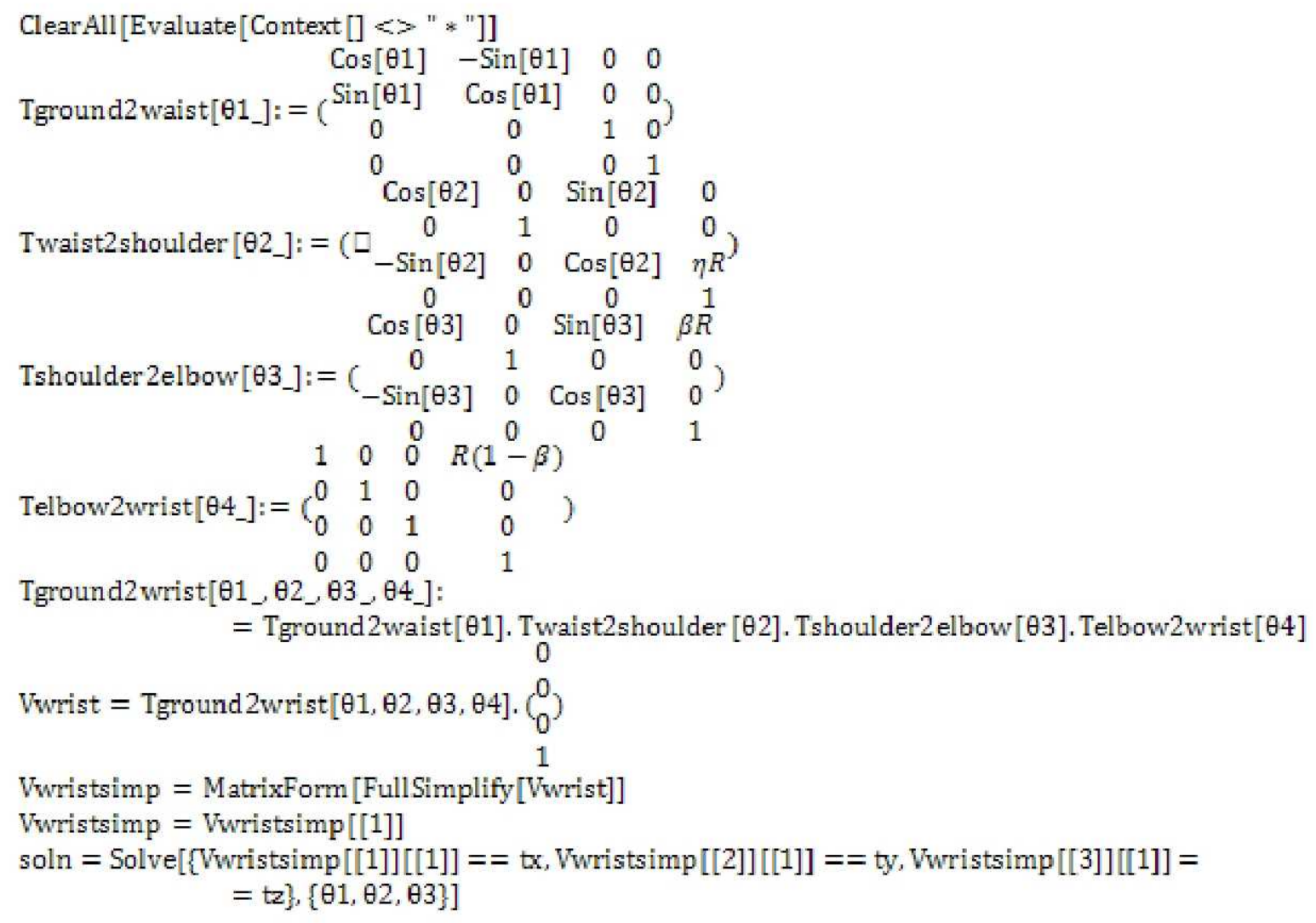




\section{Appendix C: Mass Properties and Geometry}

The mass of each body is recorded in Table 5 .

Table 5. Mass

\begin{tabular}{|l||c|}
\hline Body & $\begin{array}{c}\text { Mass } \\
(\mathrm{kg})\end{array}$ \\
\hline King Post & 88.8735 \\
\hline Arm & 81.7850 \\
\hline Forearm & 32.7304 \\
\hline Lifting Link & 3.5974 \\
\hline Payload & 150.0 \\
\hline
\end{tabular}

Moments of inertia and products of inertia, referred to collectively as inertia scalars, are with respect to the mass center of each body, and are reported in Table 6 for the reference configuration illustrated in Fig. 7.

Table 6. Inertia Scalars

\begin{tabular}{|l|r|r|r|r|r|r|}
\hline Body & $\begin{array}{c}\mathrm{I}_{11} \\
\left(\mathrm{~kg}-\mathrm{m}^{2}\right)\end{array}$ & $\begin{array}{c}\mathrm{I}_{22} \\
\left(\mathrm{~kg}-\mathrm{m}^{2}\right)\end{array}$ & $\begin{array}{c}\mathrm{I}_{33} \\
\left(\mathrm{~kg}-\mathrm{m}^{2}\right)\end{array}$ & $\begin{array}{c}\mathrm{I}_{12} \\
\left(\mathrm{~kg}-\mathrm{m}^{2}\right)\end{array}$ & $\begin{array}{c}\mathrm{I}_{23} \\
\left(\mathrm{~kg}-\mathrm{m}^{2}\right)\end{array}$ & $\begin{array}{c}\mathrm{I}_{13} \\
\left(\mathrm{~kg}-\mathrm{m}^{2}\right)\end{array}$ \\
\hline \hline King Post & 198.7825 & 230.1985 & 32.7615 & -1.2030 & -2.2441 & -33.2139 \\
\hline Arm & 33.3894 & 165.9743 & 133.8360 & -0.9035 & 1.5218 & 24.8087 \\
\hline Forearm & 4.8377 & 60.1935 & 55.5114 & -0.0067 & -0.0010 & 9.2589 \\
\hline Lifting Link & 0.5606 & 0.5541 & 0.0076 & 0 & 0.0025 & 0.0052 \\
\hline Payload & 33.6794 & 27.0725 & 13.7197 & 0 & 0 & 0 \\
\hline
\end{tabular}

For each body, the position vector from a point on the axis of the inboard joint to the mass center is reported in Table 7, followed by the position vector from the mass center to a point on the axis of the outboard joint. Numerical values are given for the reference configuration illustrated in Fig. 7. In the case of the king post, the inboard and outboard joints are, respectively, the waist joint and the shoulder joint. The inboard and outboard joints for the arm are, respectively, the shoulder joint and the elbow joint. The designation of inboard and outboard joints for the remaining bodies follows similarly. The point on the axis of the waist joint is fixed in both an inertial reference frame and the king post. The point on the axis of the shoulder joint is fixed in both the king post and the arm. Other points on each joint axis are likewise fixed in each of two adjacent bodies.

Table 7. Locations of mass centers and joints

\begin{tabular}{|l|c|c|c||c|c|c|}
\hline Body & \multicolumn{2}{|c||}{ Inboard joint to mass center } & \multicolumn{3}{c|}{ Mass center to outboard joint } \\
& $\begin{array}{c}\mathrm{r}_{1} \\
(\mathrm{~m})\end{array}$ & $\begin{array}{c}\mathrm{r}_{2} \\
(\mathrm{~m})\end{array}$ & $\begin{array}{c}\mathrm{r}_{3} \\
(\mathrm{~m})\end{array}$ & $\begin{array}{c}\mathrm{r}_{1} \\
(\mathrm{~m})\end{array}$ & $\begin{array}{c}\mathrm{r}_{2} \\
(\mathrm{~m})\end{array}$ & $\begin{array}{c}\mathrm{r}_{3} \\
(\mathrm{~m})\end{array}$ \\
\hline \hline King Post & -0.6801 & -0.0200 & 1.1254 & 0.6801 & 0.0200 & 2.5230 \\
\hline Arm & 0.5641 & -0.0214 & 0.7826 & 3.2145 & 0.0214 & -0.7840 \\
\hline Forearm & 0.8345 & -0.0001 & 0.4422 & 2.9221 & 0.0001 & -0.4110 \\
\hline Lifting Link & 0 & 0 & -0.2730 & 0 & 0 & -0.2730 \\
\hline Payload & 0 & 0 & -0.6858 & & & \\
\hline
\end{tabular}




\section{References}

${ }^{1}$ Adinolfi, P. J.; and, Heinz, F. A. Jr., "Design Study of Special Purpose Systems For the Lunar Surface." NASA CR-61077, April 30, 1965.

${ }^{2}$ Eagle Engineering, "Lunar Surface Construction and Assembly Equipment Study: Lunar Base Systems Study Task 5.3." NASA CR-172105, September, 1988.

${ }^{3}$ NASA, "NASA’s Exploration Systems Architecture Study, Final Report." NASA TM-2005-214062, 2005.

${ }^{4}$ Shapiro, H. I.; et. al., Cranes and Derricks. McGraw-Hill Inc., 1980, 2nd ed. 1991.

${ }^{5}$ Doggett, William R.; King, Bruce D.; Jones, Thomas C.; Dorsey, John T.; and Mikulas,Martin M., "Design and Field Test of a Mass Efficient Crane For Lunar Payload Handling and Inspection - The Lunar Surface Manipulation System." Presented at the AIAA Space 2008 Conference and Exposition, 9 - 11 September 2008, San Diego, California. Available as AIAA- 2008-7635.

${ }^{6}$ Dorsey, John T.; Mikulas, Martin M.; and Doggett, William R., "Preliminary Structural Design Considerations and Mass Efficiencies for Lunar Surface Manipulator Concepts". Presented at the AIAA Space 2008 Conference and Exposition, 9 - 11 September 2008, San Diego, California. Available as AIAA-2008-7916.

${ }^{7}$ Doggett, William, Dorsey, John, Collins, Tim, King, Bruce and Mikulas, Martin, "A Versatile Lifting Device for Lunar Surface Payload handling, Inspection \& Regolith Transport Operations”, STAIF-2008, Feb. 10-14 2008.

${ }^{8}$ Craig, John J. Introduction to Robotics Mechanics and Control, $2^{\text {nd }}$ ed., Addison-Wesley Publishing Company, New York, 1989, Chap. 2.

${ }^{9}$ Kane, T. R., and Levinson, D. A., Dynamics: Theory and Applications, McGraw-Hill, New York, 1985.

${ }^{10}$ Kane, T. R., and Levinson, D. A., Autolev 4 User's Manual, OnLine Dynamics, Inc., Sunnyvale, CA, 2005.

${ }^{11}$ Bryson, A. E., and Ho, Y.-C., Applied Optimal Control, Hemisphere Publishing Corporation, Washington D. C., 1975.

${ }^{12}$ Kierzenka, J., and Shampine, L. F., "A BVP Solver Based on Residual Control and the Matlab PSE," ACM Transactions on Mathematical Software, Vol. 27, No. 3, 2001, pp. 299-316.

${ }^{13}$ Gill, P. E., Murray, W. and Saunders, M. A., User's Guide for SNOPT Version 7: Software for Large-Scale Nonlinear Programming. February 12, 2006. 